\title{
Foramen Secundum
}

National Cancer Institute

\section{Source}

National Cancer Institute. Foramen Secundum. NCI Thesaurus. Code C34179.

An opening located in the septum primum that allows blood to flow directly between the right and left atrium, and which serves as a precursor to the interatrial septum. 